\title{
A PRELIMINARY CHECKLIST OF SPIDERS (ARANEAE: ARACHNIDA) IN Chinnar Wildlife SANCtuary, Western GHATS, INDIA
}

\author{
C.K. Adarsh ${ }^{1}$ \& P.O. Nameer ${ }^{2}$ \\ ${ }^{1,2}$ Centre for Wildlife Sciences, College of Forestry, Kerala Agricultural University, Thrissur, Kerala 680656, India \\ ${ }^{1}$ adarshckcof09@gmail.com, ${ }^{2}$ nameer.po@kau.in (corresponding author)
}

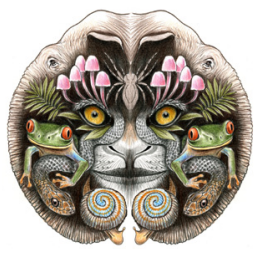

ISSN 0974-7907 (Online) ISSN 0974-7893 (Print)

OPEN ACCESS

\begin{abstract}
A preliminary study was conducted to document spider diversity in Chinnar Wildlife Sanctuary, Idukki District, Kerala State in southern India. The study was conducted from October to November 2012. A total of 101 species of spiders belonging to 65 genera from 29 families were identified from the sanctuary. This accounted for $6.98 \%$ of Indian spider species, $17.81 \%$ of Indian spider genera and $48.33 \%$ of the spider families of India. The dominant families were Lycosidae (11 species) and Araneidae (10). Two endemic genera of Indian spiders such as Annandaliella and Neoheterophrictus were found at Chinnar, each representing one species each, and belonging to the family Theraphosidae. A guild structure analysis of the spiders revealed seven feeding guilds such as orb weavers, stalkers, ground runners, foliage runners, sheet web builders, space web builders and ambushers.
\end{abstract}

Keywords: Endemism, guild structure, Idukki, Kerala.

Spiders serve an important ecological role in the ecosystem functioning as they are predatory in nature and feed on several other insects and even small vertebrates. Spiders are also considered as indicators of the health of the ecosystem (Mathew et al. 2009). A world without spiders would have serious problems affecting the whole food chain and cause an imbalance in the ecosystem; however, their study has always remained neglected. And there exist lots of misunderstanding about this most ubiquitous and diverse groups of organisms. All the spiders are regarded as poisonous creatures, and the general perception about them among the people are negative. But the fact is that very few spiders are actually poisonous and harmful to human beings (Mathew et al. 2009). However, the services these creature do to mankind by way of controlling pest species have been well documented (Riechert \& Lockley 1984; Tanaka 1989; Bishop \& Riechert 1990). Being a less charismatic species and the scarcity of biologists studying spiders, studies on the spiders of India in general and Western Ghats in particular remain scant. One of the earliest taxonomical documentations of the spider diversity of the country was by Pocock $(1895,1899,1900)$. The important studies on spider diversity of the Western Ghats were by Hirst (1909), Gravely (1915, 1935), Sherriff (1919, 1920 a,b,c), Sinha (1951), Subramanian (1955), Smith (2004), and Sugumaran et al. (2005).

Some of the recent published work on spider diversity of the Western Ghats are as follows: Sudhikumar et al. (2005a), reported 94 species from the Kuttanad rice ecosystem, central Kerala Sudhikumar et al. (2005b), also reported 72 species from Mannavan Shola, which is part of Anamudi Shola National Park. Sebastian et al. (2005) reported 51 species from Mangalavanam Bird Sanctuary,

DOI: http://dx.doi.org/10.11609/jott.2740.8.4.8703-8713 | ZooBank: urn:Isid:zoobank.org:pub:3C3B994D-DE71-431C-B6AA-ABF7907F53DF
$\begin{array}{ll}\text { Editor: Manju Siliwal, WILD, Coimbatore, India. } & \text { Date of publication: } 26 \text { April } 2016 \text { (online \& print) }\end{array}$

Manuscript details: Ms \# 2740 | Received 11 March 2015 | Final received 01 April 2016 | Finally accepted 05 April 2016

Citation: Adarsh, C.K. \& P.O. Nameer (2016). A preliminary checklist of spiders (Araneae: Arachnida) in Chinnar Wildlife Sanctuary, Western Ghats, India. Journal of Threatened Taxa 8(4): 8703-8713; http://dx.doi.org/10.11609/jott.2740.8.4.8703-8713

Copyright: @ Adarsh \& Nameer 2016. Creative Commons Attribution 4.0 International License. JoTT allows unrestricted use of this article in any medium, reproduction and distribution by providing adequate credit to the authors and the source of publication.

Funding: Kerala Agricultural University.

Conflict of Interest: The authors declare no competing interests.

Acknowledgements: We thank the Wildlife Warden, Munnar Wildlife Division and the Assistant Wildlife Warden Chinnar Wildlife Sanctuary for the logistical support. Our sincere thanks to Dr. A.V. Sudhikumar for helping us with the confirmation of the identification of the spiders. Our thanks are due to the Dean, College of Forestry, Kerala Agricultural University for encouragement and support and R. Sreehari for the map of the study area. We also thank the anonymous reviewers and the subject editor for their critical comments which greatly improved the manuscript. 
while Jose et al. (2008) documented 147 species from Parambikulam Tiger Reserve, all from the Kerala part of the Western Ghats, except Kuttanad and Magalavanam which are coastal wetlands in central Kerala. Adarsh \& Nameer (2015) reported 86 species of spiders from the Kerala Agricultural University campus, Thrissur, Kerala.

\section{STUDY AREA}

Chinnar Wildlife Sanctuary is located $18 \mathrm{~km}$ north of Marayoor in the Marayoor and Kanthalloor Panchayaths of Devikulam Taluk in the Idukki District of Kerala State (Fig. 1). It is located between $10^{\circ} 15^{\prime}-10^{\circ} 21^{\prime} \mathrm{N}$ and $77^{\circ} 5^{\prime}-$ $77^{0} 16^{\prime} \mathrm{E}$ and has a total area of $90.44 \mathrm{~km}^{2}$. The Munnar-
Udumalpet road, SH-17 passes through the Sanctuary for $16 \mathrm{~km}$ and divides it into nearly equal portions. It is contiguous with Eravikulam National Park to the South and Indira Gandhi Wildlife Sanctuary is to the north. It forms an integral part of the $1,187 \mathrm{~km}^{2}$ block of protected forests in the Anamalai Hills, Western Ghats.

The terrain is undulating with hills and hillocks of varying heights. The altitude ranges from 400-2372 m. The sanctuary is situated in the rain shadow region and hence the area experiences a prolonged hot/dry season and fewer rainy days. The Chinnar plains are generally hot, but the higher altitudes are cool. The major rainfall season is during the north-east monsoons occurring

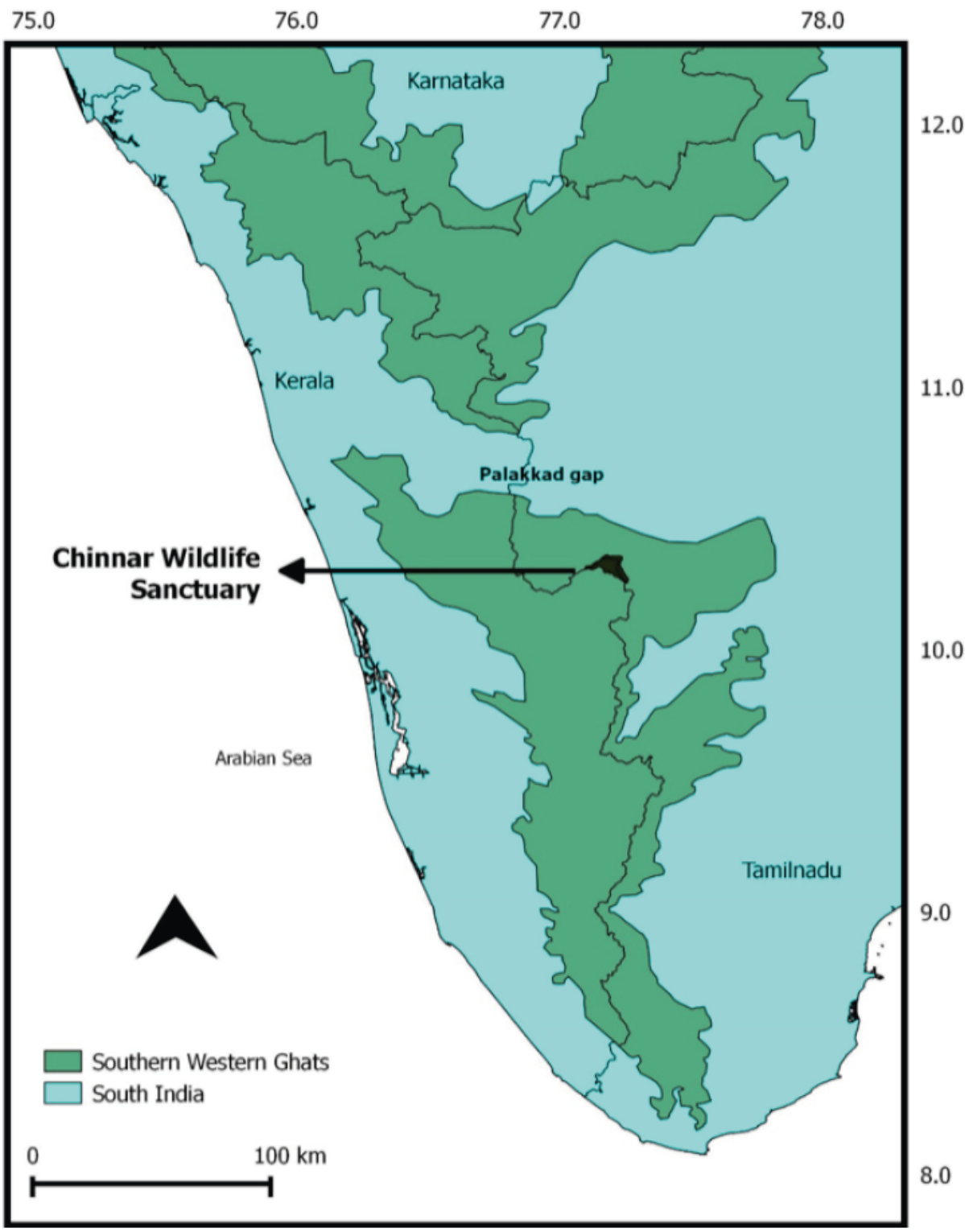

Figure 1. Location map of Chinnar Wildlife Sanctuary, southern Western Ghats 
from October to December. The rainy days in a year range from between $30-40$ days which account for about 300-500 mm rainfall in Chinnar. But the higher altitudes areas like Olikkudy and Mangappara receive rain during both the north-east and south-west monsoons with comparatively much higher rainfall. The recorded lowest temperature is $12^{\circ} \mathrm{C}$ and the highest is $38^{\circ} \mathrm{C}$.

The vegetation shows an entire spectrum ranging from temperate shola to dry scrub of the arid plains. The vegetation of the sanctuary can be broadly classified into the following types according to Champion \& Seth (1968). They are southern tropical thorn forest (scrub jungle), southern dry mixed deciduous forest (dry deciduous forest), southern moist mixed deciduous forest (moist deciduous forest), tropical riparian fringing forest (riparian forest), southern montane wet temperate forest (montane shola forest) and southern montane wet grassland (grasslands).

\section{MethodS}

The study was conducted from October to November 2012. Bushes, tree trunks, ferns, forest floor, foliage and grasslands were all searched for spiders and collected by handpick method as suggested by Tikader (1987). The identification of spiders was done following Tikader (1970, 1977, 1980, 1982, 1987), Koh (1996), Murphy \& Murphy (2000), Sebastian \& Peter (2009). The checklist of the spiders of Chinnar Wildlife Sanctuary is prepared and is presented in this paper. The taxonomy and nomenclature followed is as per the world spider catalogue (Platnick 2014).

\section{RESULTS AND DISCUSSION}

Out of 1447 spiders recorded from India (Siliwal \& Molur 2005, 2007), 101 species belonging to 65 genera of 29 families were recorded during the present study (Appendix 1). This accounts for $6.98 \%$ of the total species of spiders and $48.33 \%$ of the total spider families recorded in India. Stegodyphus sarasinorum (Image 10) was found to be the most abundant species followed by Hersilia savignyi (Image 16), Palpimanus gibbulus (Image 33), Cyclosa hexatuberculata (Image 2), Selenops radiates (Image 48), Heteropoda hampsoni (Image 49), Gnaphosa kailana (Image 13). The most speciose spider family of Chinnar was Lycosidae (11 species) followed by Araneidae (10), Salticidae (8 species), Tetragnathidae (8 species), Oxyopidae (6 species) and Theridiidae (6 species).

Out of the 101 species identified from Chinnar Wildlife Sanctuary, 34 species are endemic to India (Appendix 1). The spiders of Chinnar are spread across 65
Orb weavers

- Stalkers

Ambushers

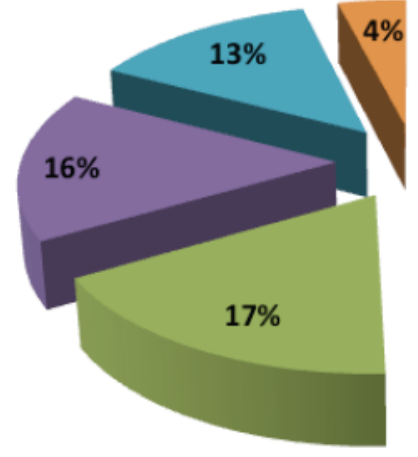

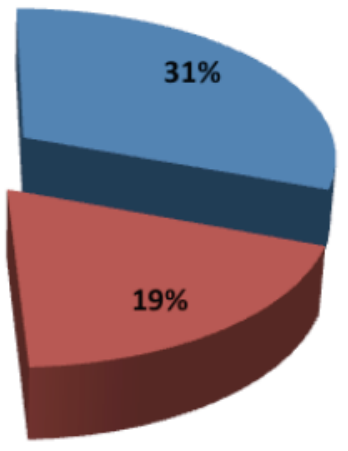

- Space web builders

Foliage runners

Sheet web builders
Figure 2. Guild structure of spiders recorded from Chinnar Wildlife Sanctuary

genera, of which two are endemic to India. The endemic genera of spiders seen at Chinnar are Annandaliella and Neoheterophrictus (Image 65), and belonging to the family Theraphosidae.

The spiders of Chinnar Wildlife Sanctuary can be divided into seven feeding guilds based on the foraging behavior (Uetz et al.1999). They are the orb weavers, stalkers, ground runners, foliage runners, sheet web builders, space web builders and ambushers. The dominant guild was of the ground runners with 26 species and is followed by orb weavers (23 species), stalkers (14 species), ambushers (13 species), space web builders (12 species), foliage runners (10 species) and sheet web builders ( 3 species) (Table 1, Fig. 2).

The present report of Latrodectus hasselti (Image 56) from Chinnar Wildlife Sanctuary is the first record of the species from Kerala State. There are only very few reports on the occurrence of Latrodectus hasselti from India. It was first reported from Pune (Simon, 1897; Pocock, 1900), later by Daniel \& Soman (1961) from Suriamal in northern Thana, Bombay, then from Vadodara (Baroda) by Siliwal \& Kumar (2001) and finally by Kumar \& Siliwal (2005) from Coimbatore, Tamil Nadu.

\section{CONCLUSION}

A preliminary checklist on the spiders of the Chinnar Wildlife Sanctuary, Western Ghats is given in this paper. This is the first ever documentation of the spiders of Chinnar. However, this by no means is comprehensive and it only suggest the great diversity of the spider fauna of Chinnar and thus warranting more detailed and systematic exploration of the spiders of Chinnar Wildlife 
Table 1. Total number of families, genera, species and functional guilds of spiders in Chinnar Wildlife Sanctuary

\begin{tabular}{|c|c|c|c|c|}
\hline & Family & $\begin{array}{l}\text { No. of } \\
\text { genera }\end{array}$ & $\begin{array}{l}\text { No. of } \\
\text { species }\end{array}$ & Guild \\
\hline 1 & Agelenidae & 1 & 1 & Space web builders \\
\hline 2 & Araneidae & 5 & 10 & Orb weavers \\
\hline 3 & Clubionidae & 1 & 2 & Foliage runners \\
\hline 4 & Ctenidae & 1 & 3 & Ground runners \\
\hline 5 & Eresidae & 1 & 1 & Space web builders \\
\hline 6 & Filistatidae & 1 & 1 & Space web builders \\
\hline 7 & Gnaphosidae & 4 & 4 & Ground runners \\
\hline 8 & Hersiliidae & 1 & 2 & Ambushers \\
\hline 9 & Linyphiidae & 1 & 2 & Sheet web builders \\
\hline 10 & Lycosidae & 5 & 11 & Ground runners \\
\hline 11 & Miturgidae & 1 & 3 & Foliage runners \\
\hline 12 & Nephilidae & 2 & 2 & Orb weavers \\
\hline 13 & Oxyopidae & 2 & 6 & Stalkers \\
\hline 14 & Palpimanidae & 1 & 1 & Ground runners \\
\hline 15 & Philodromidae & 1 & 1 & Ambushers \\
\hline 16 & Pholcidae & 2 & 2 & Space web builders \\
\hline 17 & Pimoidae & 1 & 1 & Sheet web builders \\
\hline 18 & Pisauridae & 4 & 5 & Ambushers \\
\hline 19 & Psechridae & 1 & 1 & Space web builders \\
\hline 20 & Salticidae & 7 & 8 & Stalkers \\
\hline 21 & Scytodidae & 1 & 2 & Ground runners \\
\hline 22 & Selenopidae & 1 & 1 & Ground runners \\
\hline 23 & Sparassidae & 1 & 5 & Foliage runners \\
\hline 24 & Tetragnathidae & 3 & 8 & Orb weavers \\
\hline 25 & Theraphosidae & 3 & 3 & Ground runners \\
\hline 26 & Theridiidae & 5 & 6 & Space web builders \\
\hline 27 & Thomisidae & 5 & 5 & Ambushers \\
\hline 28 & Uloboridae & 2 & 3 & Orb weavers \\
\hline 29 & Zodariidae & 1 & 1 & Ground runners \\
\hline
\end{tabular}

Sanctuary and other protected areas too.

\section{REFERENCES}

Adarsh, C.K. \& P.O. Nameer (2015). Spiders of Kerala Agricultura University Campus, Thrissur, Kerala, India. Journal of Threatened Taxa 7(15): 8288-8295; http://dx.doi.org/10.11609/jott.2468.7.15.82888295

Bishop, L. \& S.E. Riechert (1990). Spider colonization of agro ecosystems: mode and source. Environmental Entomology 19: 1738-1745; http://dx.doi.org/10.1093/ee/19.6.1738

Champion, H.G. \& P.K. Seth (1968). A Revised Survey of the Forest Types of India. Government of India Press, Nasik, New Delhi, India, 404pp.

Daniel, J.C. \& P.W. Soman (1961). Observations on the spider Latrodectus hasseltii indicus Simon with a note on arachnidism. Journal of the Bombay Natural History Society 58(3): 823-826.
Gravely, F.H. (1915). Notes on Indian mygalomorph spiders - I. Records of Indian Museum 11: 257-287.

Gravely, F.H. (1935). Notes on Indian mygalomorph spiders - II. Records of Indian Museum 37: 69-84.

Hirst, A.S. (1909). On some new or little-known mygalomorph spiders from the oriental region and Australasia. Records of Indian Museum 3: 383-390.

Jose, S.K., A.V. Sudhikumar, S. Davis \& P.A. Sebastian (2008). Preliminary studies on the spider fauna (Arachnida: Araneae) in Parambikulam Wildlife Sanctuary in Western Ghats, Kerala, India. Journal of the Bombay Natural History Society 105(3): 264-273.

Kumar, M.G. \& M. Siliwal (2005). Range extension of Latrodectus hasselti Thorell, 1870 (Araneae: Theridiidae). Zoos' Print Journal 20(11): 2072; http://dx.doi.org/10.11609/JoTT.ZPJ.1344.2072

Koh J.K.H. (1996). A Guide to Common Singapore Spiders - Second Edition. Singapore Science Centre, Singapore.

Mathew, M.J., A.V. Sudhikumar \& K.S. Jose (2009) Natural history and bioecology, pp. 40-63. In: Sebastian, P.A. \& K.V. Peter (eds.). Spiders of India. Universities Press (India) Pvt. Ltd., 614pp.

Murphy, F. \& J. Murphy (2000). An Introduction to the Spiders of South East Asia. Malaysian Nature Society, Malaysia, 624pp.

Platnick, N.I. (2014). The World Spider Catalog, version 14.5. American Museum of Natural History http://research.amnh.org/entomology/ spiders/catalog/index. html. Accessed on 14 December 2014

Pocock, R.I. (1895). Notes on the identity of some of the types of Mygalomorphae in the collection of the British Museum. Annals and Magazine of Natural History 16(sixth series): 223-230.

Pocock, R.I. (1899). Diagnoses of some new Indian Arachnida. Journal of the Bombay Natural History Society 12: 744-753.

Pocock, R.I. (1900). Great Indian spiders - the genus Poecilotheria: its habits, history and species. Journal of the Bombay Natural History Society 13: 121-133.

Riechert, S.E. \& T. Lockley (1984). Spiders as biological control agents. Annual Review of Entomology 29: 299-320.

Sebastian, P.A., S. Murugesan, M.J. Mathew, A.V. Sudhikumar \& E. Sunish (2005). Spiders in Mangalavanam, an ecosensitive mangrove forest in Cochin, Kerala, India (Araneae). European Arachnology (Suppl. No. 1): 315-318.

Sebastian, P.A. \& K.V. Peter (2009). Spiders of India. Universities Press (India) Pvt. Ltd., 614pp.

Sherriff, W.R. (1919). A contribution to the study of South Indian Arachnology I. Annals and Magazine of Natural History 9: 220-253.

Sherriff, W.R. (1927a). A contribution to the study of South Indian Arachnology II. Annals and Magazine of Natural History 9: 533-542.

Sherriff, W.R. (1927b). A contribution to the study of South Indian Arachnology III. Annals and Magazine of Natural History 10 (2): 177-181.

Sherriff, W.R. (1927c). A contribution to the study of South Indian Arachnology IV. Annals and Magazine of Natural History 10(2): 233-249.

Siliwal, M. \& D. Kumar (2001). Rare sighting of poisonous spider Latrodectus hasseltii indicus Simon (Araneae: Theridiidae) in a cotton field in Baroda District, Gujarat. Current Science 81(9): 1170.

Siliwal, M., S. Molur \& B.K. Biswas (2005). Indian spiders (Arachnida: Araneae): updated checklist 2005. Zoos' Print Journal 20(10): 19992049; http://dx.doi.org/10.11609/JoTT.ZPJ.1283.1999-2049

Siliwal, M. \& S. Molur (2007). Checklist of spiders (Arachnida: Araneae) of South Asia including the 2006 update of Indian spider checklist. Zoos' Print Journal 22(2): 2551-2597; http://dx.doi.org/10.11609/ JoTT.ZPJ.1509.2551-97

Simon, E. (1897). Arachnides recueillis par M.M. Maindron à Mascate, en octobre 1896. Bulletin du Museum (national)d'histoire naturelle 95-98pp.

Sinha, T.B. (1951). On the collection of lycosid spiders in the Zoological Survey of India. Records of Indian Museum 48: 9-52.

Smith, A.M. (2004). A new species of the arboreal theraphosid, genus Poecilotheria, from southern India (Araneae, Mygalomorphae, Theraphosidae) with notes on its conservation status. Journal of British Tarantula Society 19: 48-61. 
Appendix 1. Checklist of spiders of Chinnar Wildlife Sanctuary, Kerala.

Family Agelenidae C.L. Koch, 1837

1. Agelena inda Simon, $1897 *$ (Image 1)

Family Araneidae Simon, 1895

2. Araneus bilunifer Pocock, $1900 *$

3. Araneus sp.

4. Cyclosa hexatuberculata Tikader, 1982* (Image 2)

5. Cyclosa spirifera Simon, 1889* (Image 3)

6. Cyrtarachne gravelyi Tikader, 1961 (Image 4)

7. Cyrtarachne sp. (Image 64)

8. Cyrtophora bidenta Tikader, 1970*

9. Cyrtophora cicatrosa (Stoliczka, 1869)

10. Cyrtophora citricola (Forskal, 1775) (Image 5)

11. Gasteracantha geminata (Fabricius, 1798) (Image 6)

Family Clubionidae Wagner, 1887

12. Clubiona drassodes O. P. Cambridge, 1874 (Image 7)

13. Clubiona $s p$.

Family Ctenidae Keyserling, 1877

14. Ctenus cochinensis Gravely, 1931* (Image 8)

15. Ctenus indicus Gravely, 1931* (Image 9)

16. Ctenus sp. (Image 65)

Family Eresidae Koch, 1851

17. Stegodyphus sarasinorum Karsch, 1891 (Image 10)

Family Filistatidae Ausserer, 1867

18. Filistata rufa Caporiacco, 1934* (Image 11)

Family Gnaphosidae Pocock, 1898

19. Drassodes carinivulvus Caporiacco, 1934* (Image 12)

20. Gnaphosa kailana Tikader, 1966* (Image 13)

21. Poecilochroa barmani Tikader, 1982* (Image 14)

22. Zelotes ashae Tikader \& Gajbe, 1976* (Image 15)

Family Hersiliidae Thorell, 1870

23. Hersilia savignyi Lucas, 1836 (Image 16)

24. Hersilia sp. (Image 17)

Family Linyphiidae Blackwall, 1859

25. Linyphia perampla O.P. Cambridge, $1885^{*}$ (Image 18)

26. Linyphia sp.

Family Lycosidae Sundevall, 1833

27. Evippa banarensis Tikader and Malhotra, 1980* (Image 19)

28. Hippasa greenalliae (Blackwall, 1867) (Image 20)

29. Hippasa holmerae Thorell, 1895* (Image 21)

30. Lycosa barnesi Gravely, 1924* (Image 22)

31. Lycosa bistriata Gravely, 1924

32. Lycosa tista Tikader, 1970* (Image 23)

33. Lycosa sp 1.

34. Lycosa sp 2

35. Pardosa pseudoannulata Bosenberg and Strand, 1906) (Image 24)

36. Pardosa sumatrana (Thorell, 1890) (Image 25)

37. Wadicosa quadrifera (Gravely, 1924) (Image 26)

Family Miturgidae Simon, 1885

38. Cheiracanthium danieli Tikader, 1975*

39. Cheiracanthium melanostomum (Thorell, 1895)

40. Cheiracanthium sp. (Image 66)

Family Nephilidae Simon, 1894

41. Nephila pilipes (Fabricius, 1793) (Image 27)

42. Nephilengys malabarensis (Walckenaer, 1842) (Image 28)

Family Oxyopidae Thorell, 1870

43. Oxyopes birmanicus Thorell, 1887 (Image 29)

44. Oxyopes javanus Thorell, 1887 (Image 30)

45. Oxyopes shweta Tikader, 1970 (Image 31)

46. Peucetia viridana (Stoliczka, 1869) (Image 32)

47. Oxyopes sp. 1

48. Oxyopes sp. 2

Family Palpimanidae Thorell, 1870

49. Palpimanus gibbulus Dufour, 1820 (Image 33)

Family Philodromidae Thorell, 1870

50. Tibellus elongatus Tikader, 1960* (Image 34)
Family Pholcidae C. L. Koch, 1851

51. Artema atlanta Walckenaer, 1837 (Image 35)

52. Pholcus phalangioides (Fuesslin, 1775) (Image 36)

Family Pimoidae Wunderlich, 1986

53. Pimoa indiscrete Hormiga, 1994* (Image 37)

Family Pisauridae Simon, 1890

54. Perenethis unifasciata (Doleschall, 1859)

55. Pisaura sp. (Image 38)

56. Polyboea vulpina Thorell, 1895

57. Thalassius albocinctus (Doleschall, 1859) (Image 67)

58. Thalassius sp.

Family Psechridae Simon, 1890

59. Psechrus torvus (Cambridge, 1869) (Image 39 )

Family Salticidae Blackwall, 1841

60. Aelurillus improvises Azarkina, 2002* (Image 40)

61. Hasarius adansoni (Audouin, 1826)

62. Menemerus bivittatus (Dufour, 1831) (Image 41)

63. Phaeacius lancearius (Thorell, 1895) (Image 42)

64. Phaeacius sp.

65. Phintella vittata (C.L. Koch, 1846) (Image 43)

66. Portia fimbriata Doleschall, 1859 (Image 44)

67. Siler semiglaucus Simon, 1901 (Image 45)

Family Scytodidae Blackwall, 1864

68. Scytodes fusca Walckenaer, 1837 (Image 46)

69. Scytodes thoracica (Latreille, 1802) (Image 47)

Family Selenopidae Simon, 1897

70. Selenops radiatus Latreille, 1819 (Image 48)

Family Sparassidae Bertkau, 1872

71. Heteropoda hampsoni Pocock, 1901* (Image 49)

72. Heteropoda lentula Pocock, 1901*

73. Heteropoda leprosa Simon, 1884 (Image 50)

74. Heteropoda venatoria (Linnaeus, 1767)

75. Heteropoda sp. (Image 68)

Family Tetragnathidae Menge, 1866

76. Leucauge decorate (Blackwall, 1864)

77. Leucauge pondae Tikader, 1970* (Image 51)

78. Leucauge tessellata (Thorell, 1887)

79. Leucauge sp.

80. Opadometa fastigata Simon, 1877) (Image 52)

81. Tetragnatha andamanensis Tikader, 1977

82. Tetragnatha fletcheri Gravely, 1921*

83. Tetragnatha maxillosa Thorell, 1895 (Image 53)

Family Theraphosidae Thorell, 1870

84. Annandaliella sp.*

85. Neoheterophrictus sp.* (Image 63)

86. Poecilotheria striata Pocock, 1895* (Image 54)

Family Theridiidae Sundevall, 1833

87. Achaearanea mundula (L. Koch, 1872)

88. Argyrodes argentatus O.P. Cambridge, 1880

89. Chrysso argyrodiformis (Yaginuma, 1952) (Image 55)

90. Latrodectus hasselti Thorell, 1870 (Image 56)

91. Achaearanea sp. 1 (image 69)

92. Achaearanea sp. 2

Family Thomisidae Sundevall, 1833

93. Misumena decorata Tikader, 1980 (Image 57)

94. Runcinia roonwali Tikader, $1965^{*}$ (Image 58)

95. Synema decoratum Tikader, 1960

96. Talaus opportunus (O.P. Cambridge, 1873)* (Image 59)

97. Xysticus breviceps O.P. Cambridge, 1885* (Image 60)

Family Uloboridae Thorell, 1869

98. Miagrammopes extensus Simon, 1889* (Image 61)

99. Miagrammopes sp.

100. Uloborus danolius Tikader, 1969

Family Zodariidae Thorell, 1881

101. Asceua cingulata (Simon, 1905)* (Image 62) 


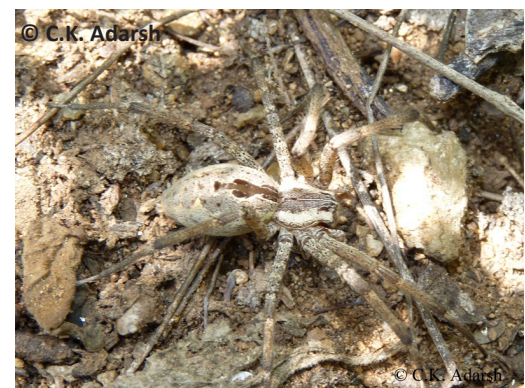

Image 1. Agelena inda

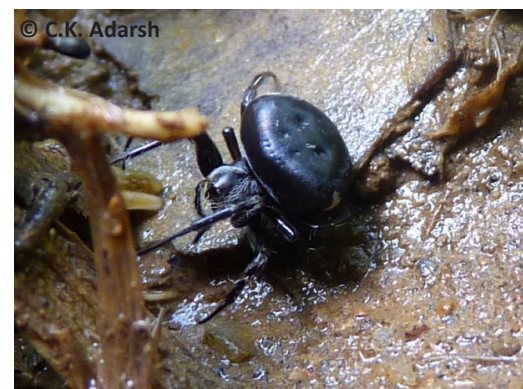

Image 4. Cyrtarachne gravelyi

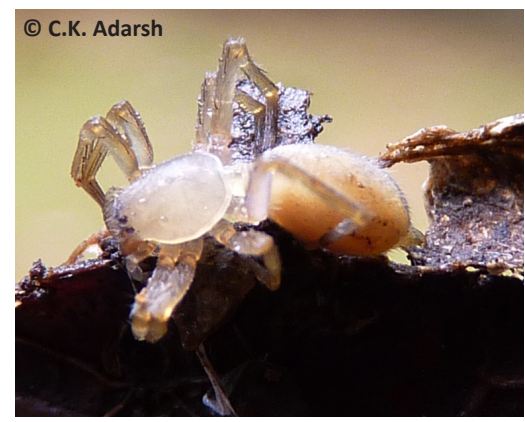

Image 7. Clubiona drassodes

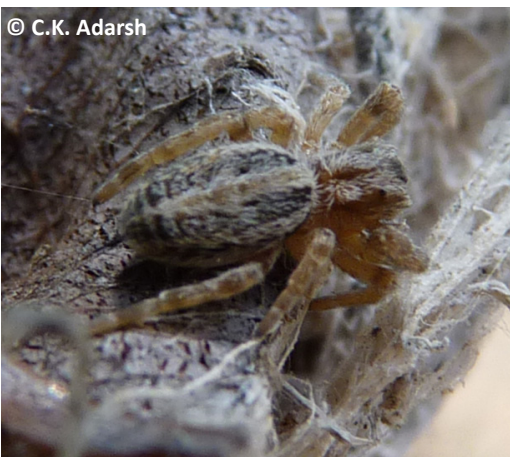

Image 10. Stegodyphus sarasinorum

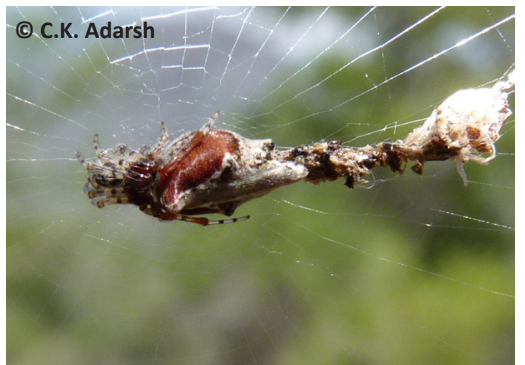

Image 2. Cyclosa hexatuberculata

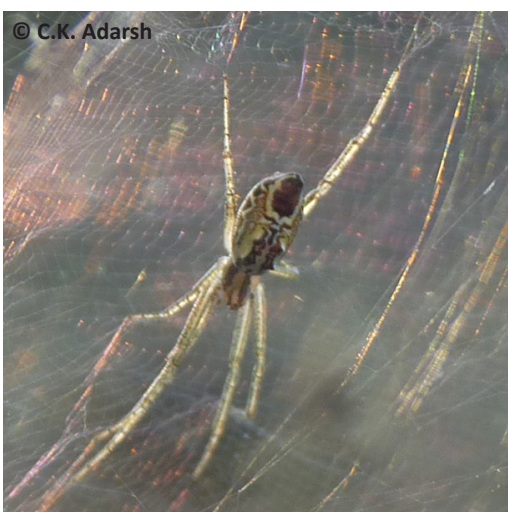

Image 5. Cyrtophora citricola

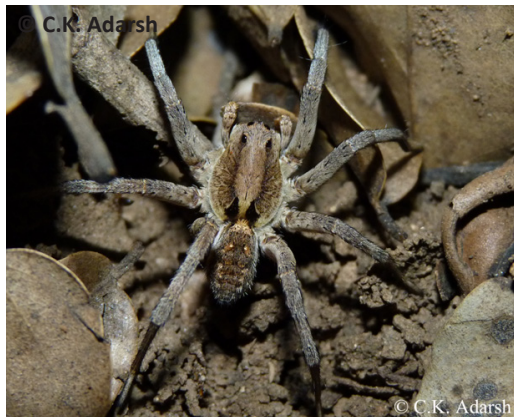

Image 8. Ctenus cochinensis

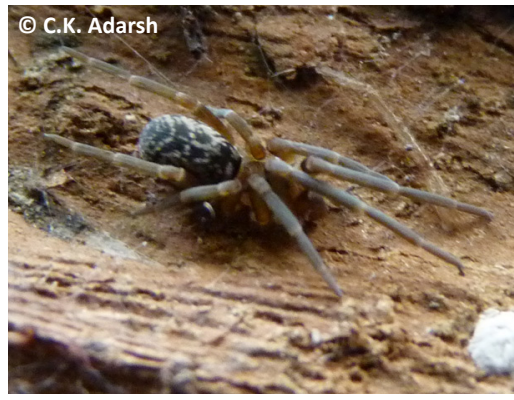

Image 11. Filistata rufa

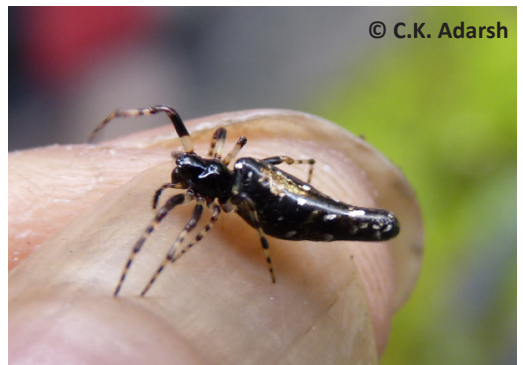

Image 3. Cyclosa spirifera

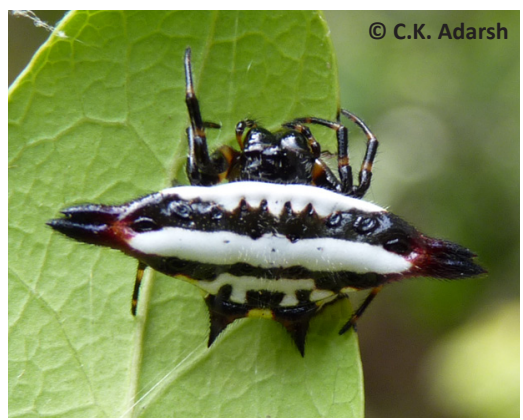

Image 6. Gasteracantha geminata

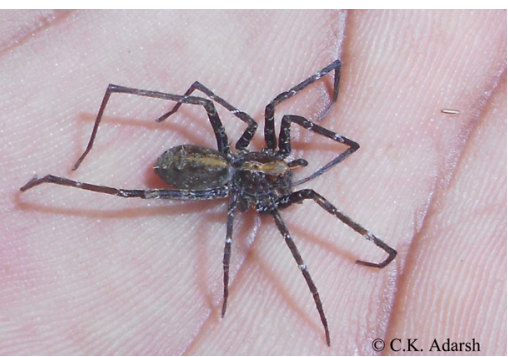

Image 9. Ctenus indicus

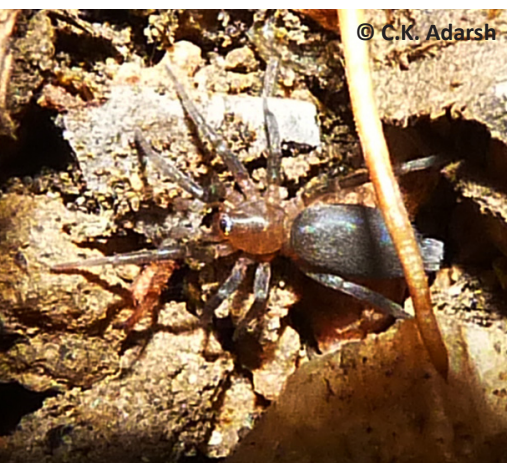

Image 12. Drassodes carinivulvus 


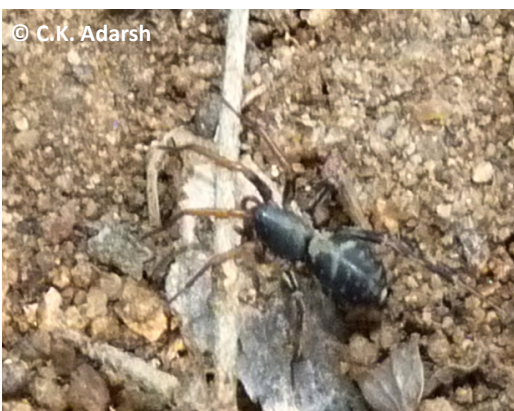

Image 13. Gnaphosa kailana

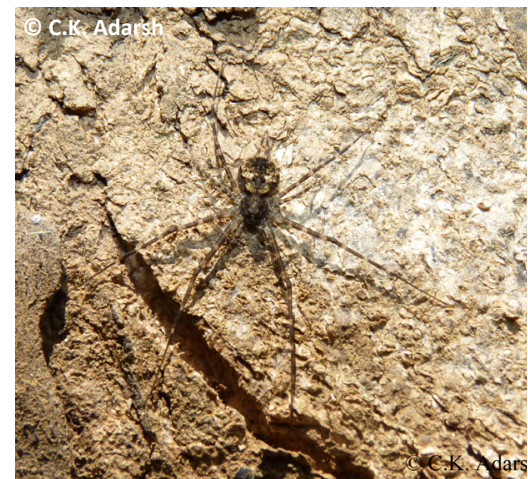

Image 16. Hersilia savignyi Lucas

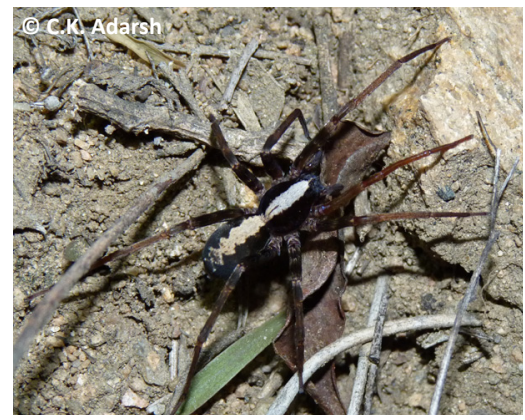

Image 19. Evippa banarensis

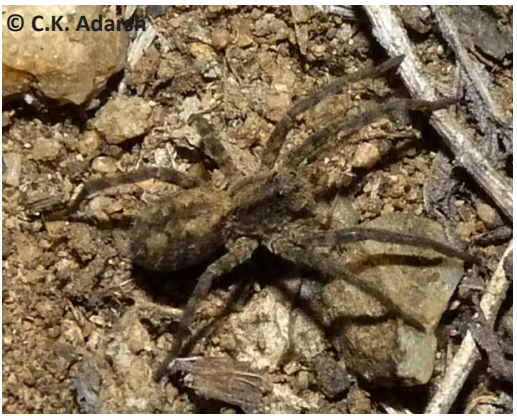

Image 22. Lycosa barnesi

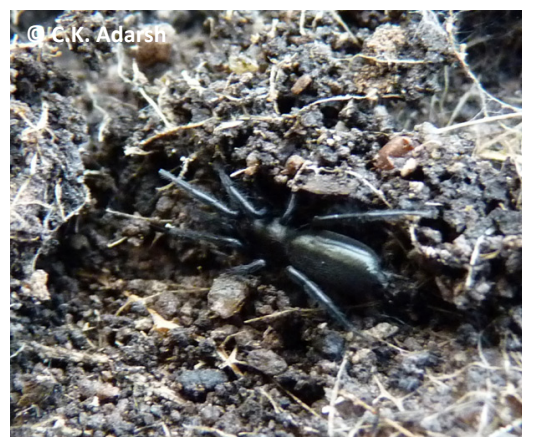

Image 14. Poecilochroa barmani

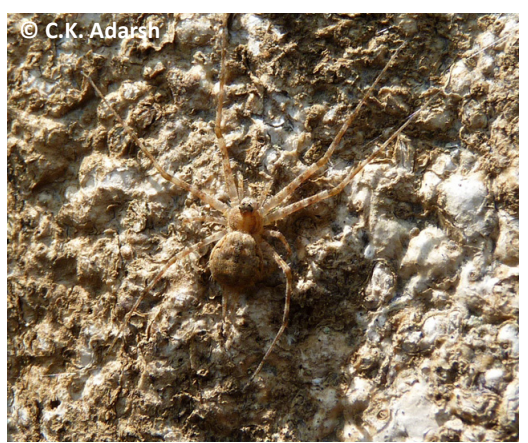

Image 17. Hersilia sp.

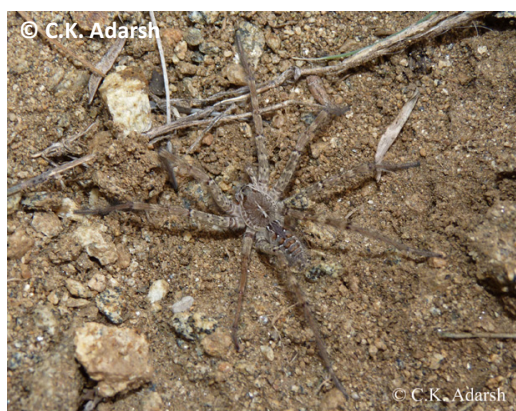

Image 20. Hippasa greenalliae

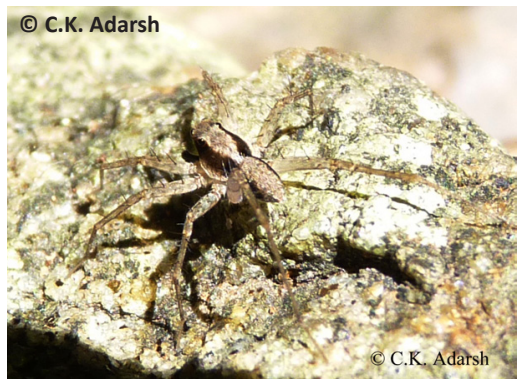

Image 23. Lycosa tista

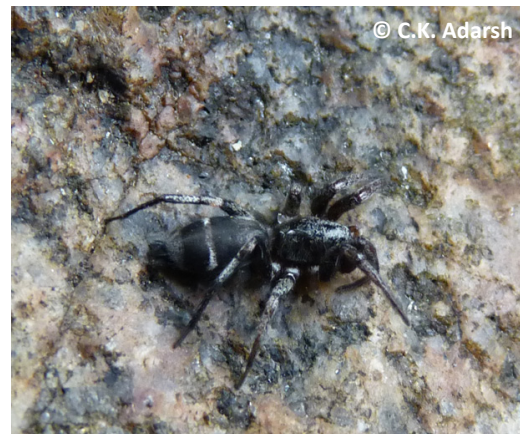

Image 15. Zelotes ashae

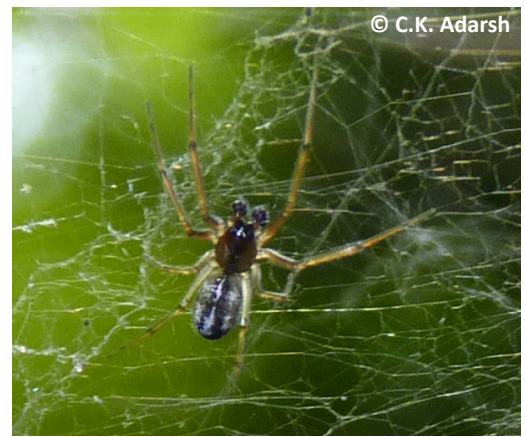

Image 18. Linyphia perampla

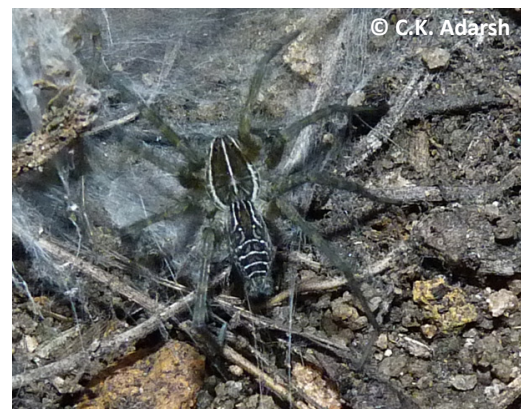

Image 21. Hippasa holomerae

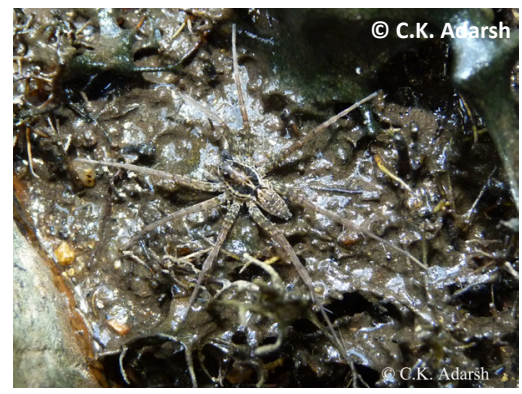

Image 24. Pardosa pseudoannulata 


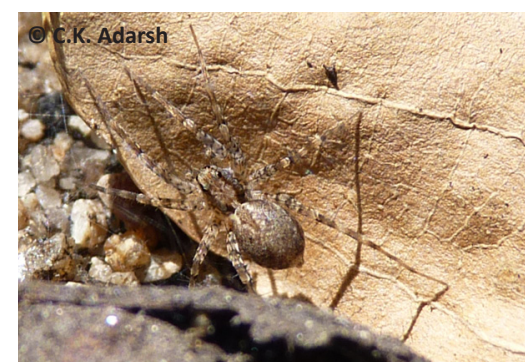

Image 25. Pardosa sumatrana

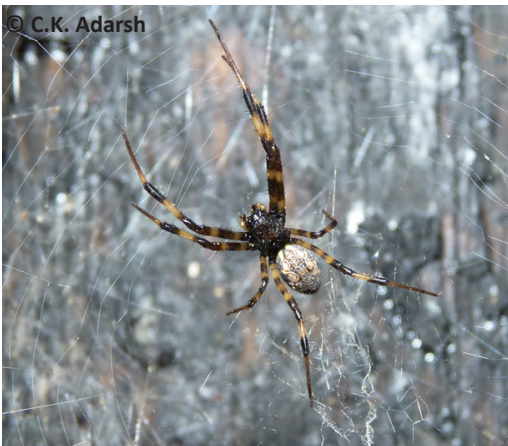

Image 28. Nephilengys malabarensis

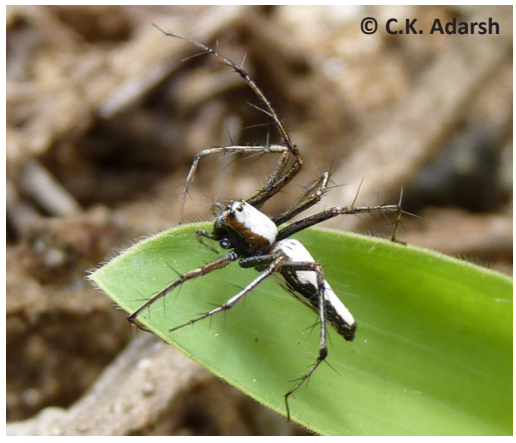

Image 31. Oxyopes shwetc

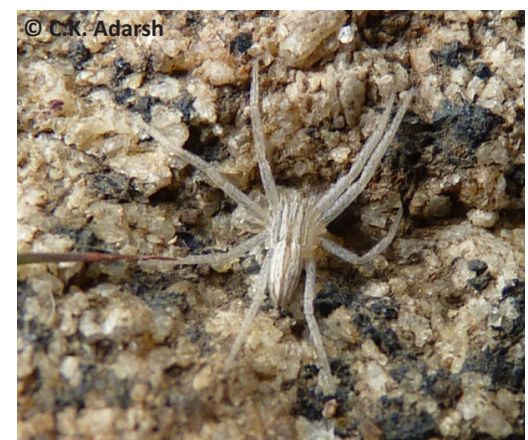

Image 34. Tibellus elongatus

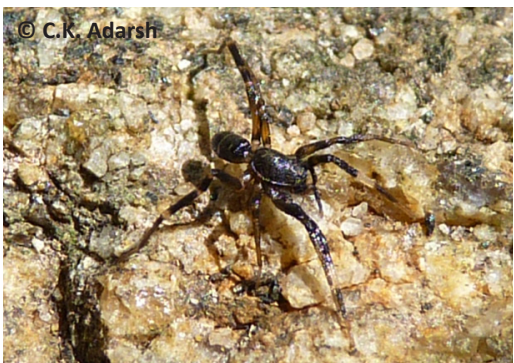

Image 26. Wadicosa quadrifera

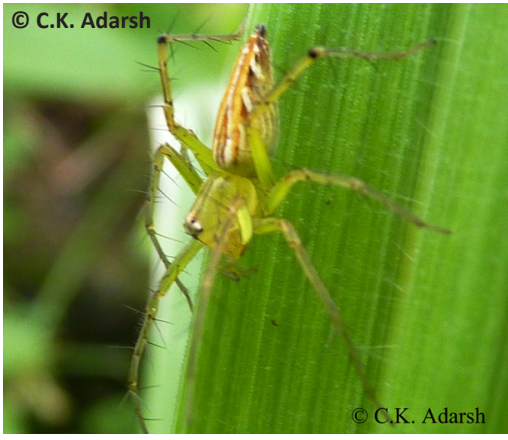

Image 29. Oxyopes biramicus

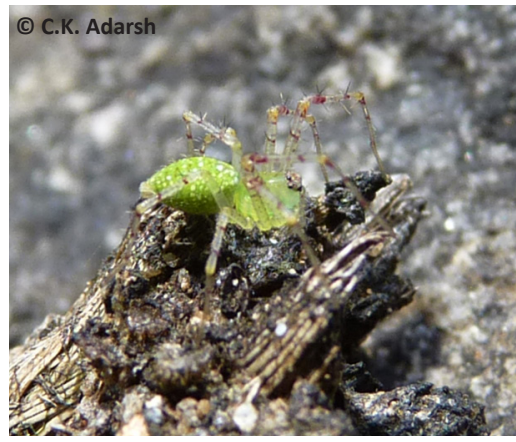

Image 32. Peucetia viridana

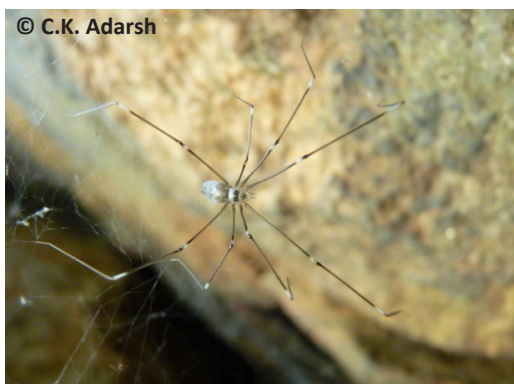

Image 35. Artema atlanta

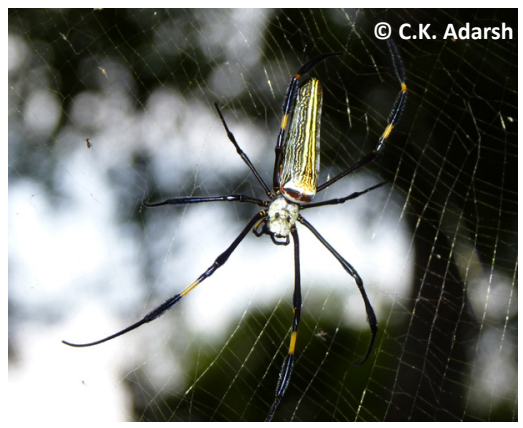

Image 27. Nephila pilipes

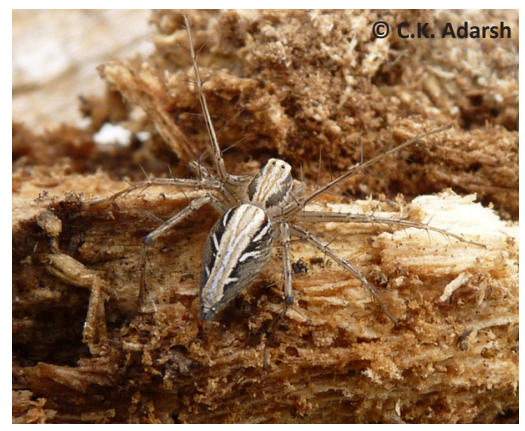

Image 30. Oxyopes javanus

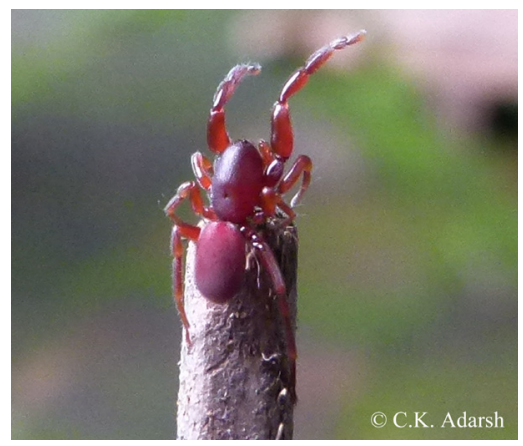

Image 33. Palpimanus gibbulus

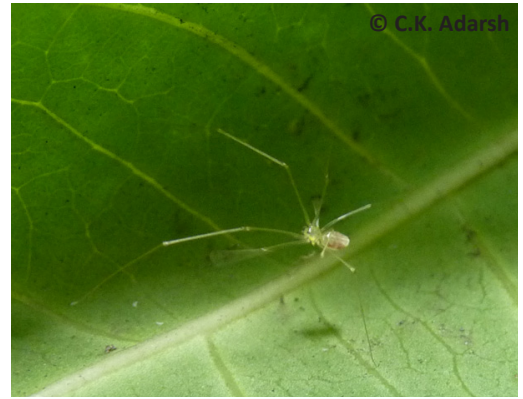

Image 36. Pholcus phalangioides 


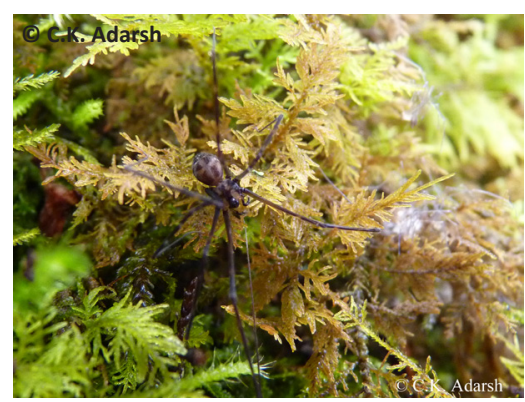

Image 37. Pimoa indiscreta

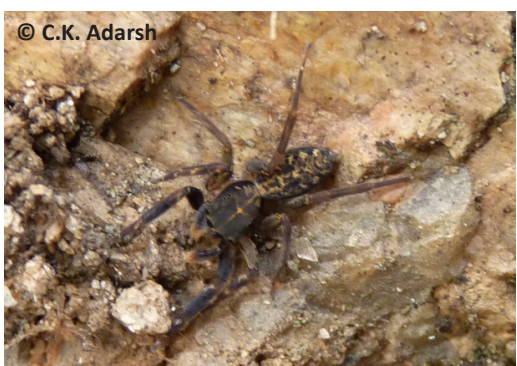

Image 40. Aelurillus improvisus

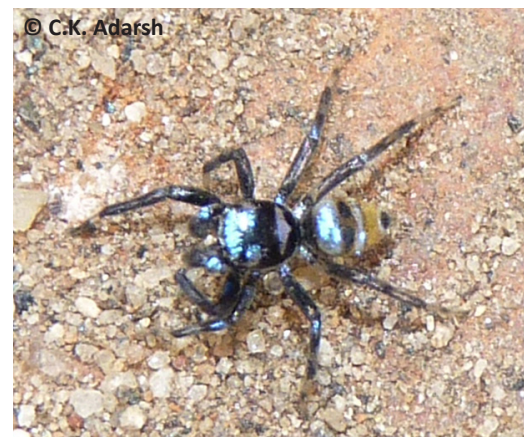

Image 43. Phintella vittato

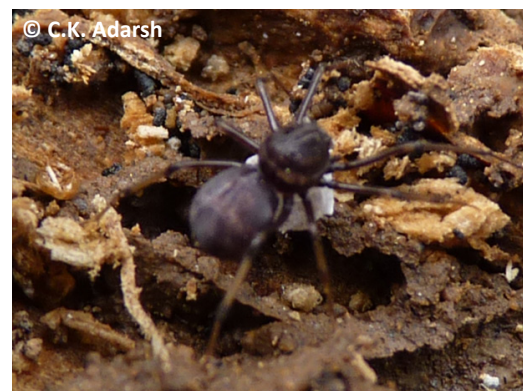

Image 46. Scytodes fusca

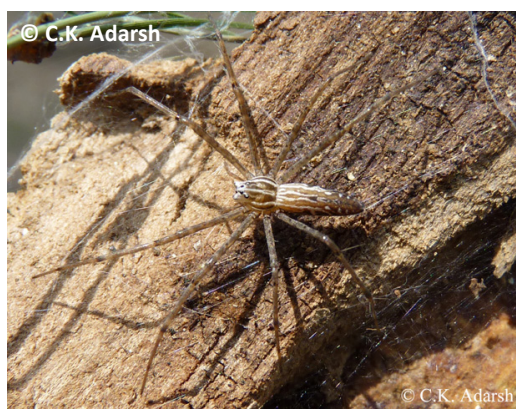

Image 38. Pisaura sp.

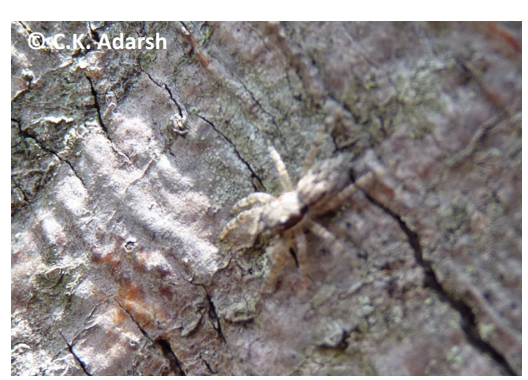

Image 41. Menemerus bivittatus

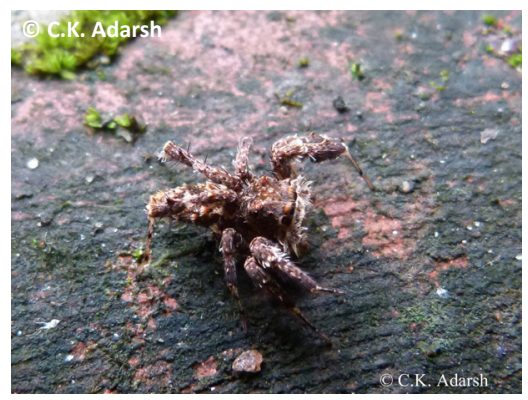

Image 44. Portia fimbriata

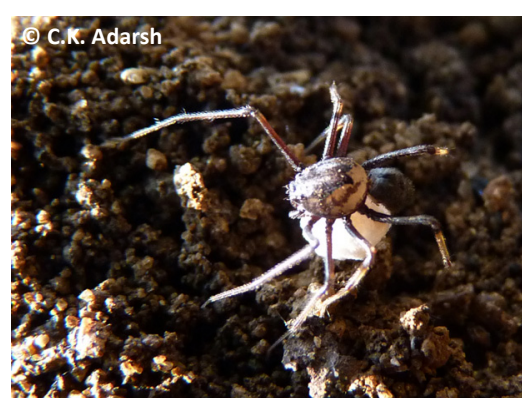

Image 47. Scytodes thoracica

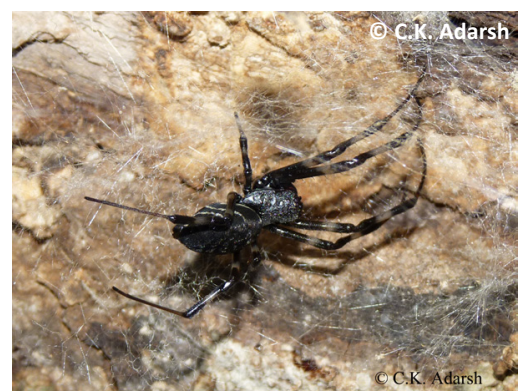

Image 39. Psechrus torvus

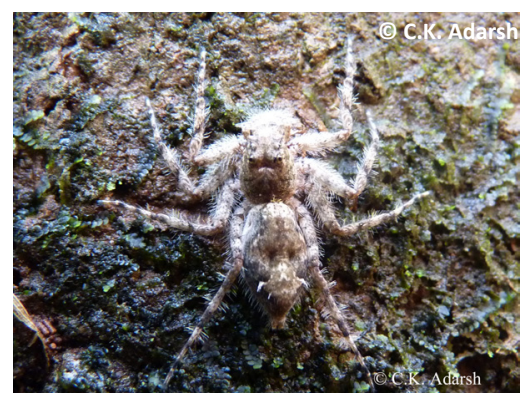

Image 42. Phaeacius lancearius

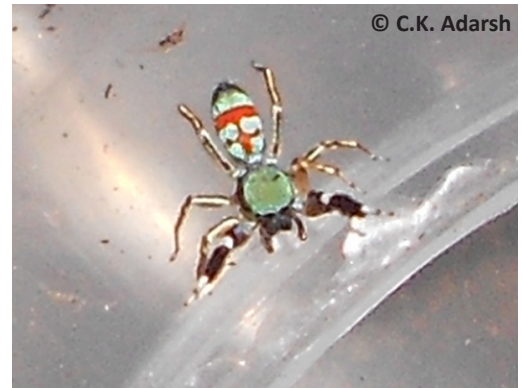

Image 45. Siler semiglaucus

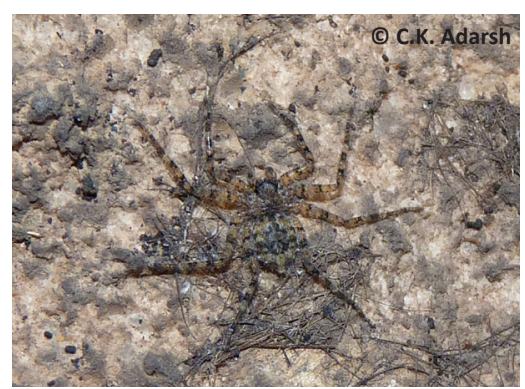

Image 48. Selenops radiatus 


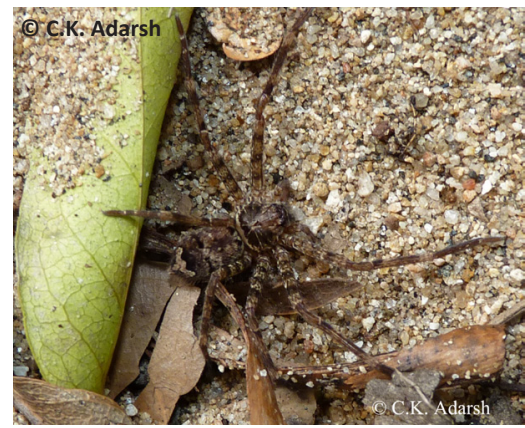

Image 49. Heteropoda hampsoni

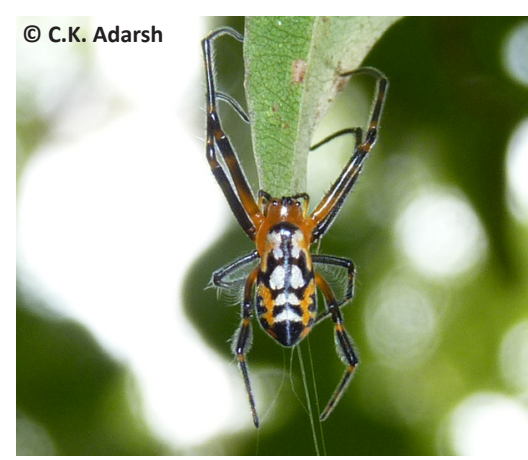

Image 52. Opadometa fastigato

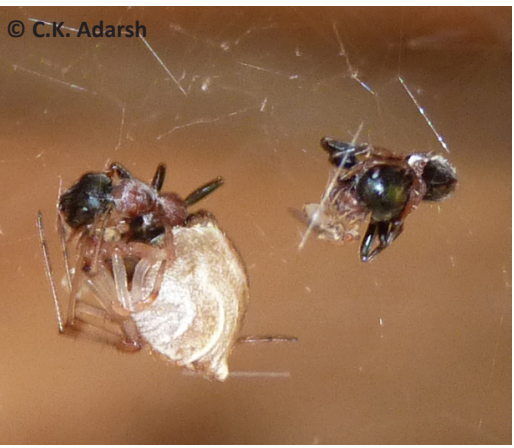

Image 55. Chrysso argyrodiformis

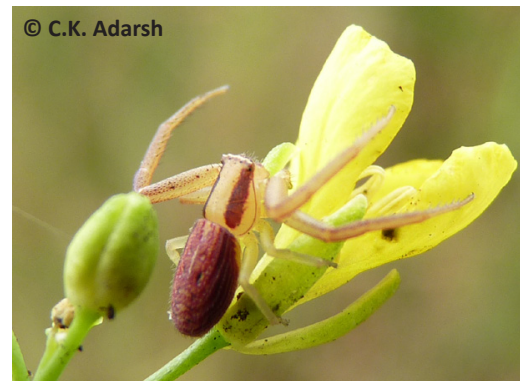

Image 58. Runcinia roonwali

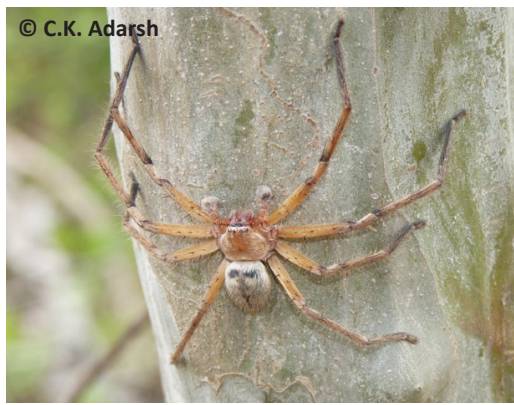

Image 50. Heteropoda leprosa

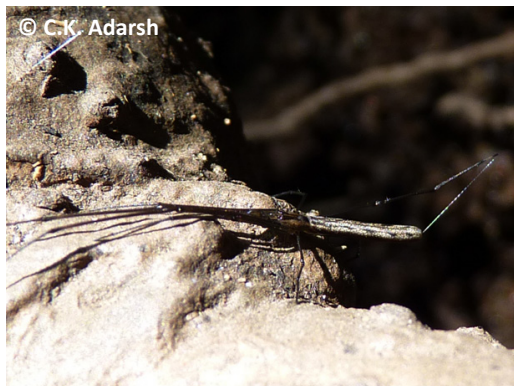

Image 53. Tetragnatha maxillosa

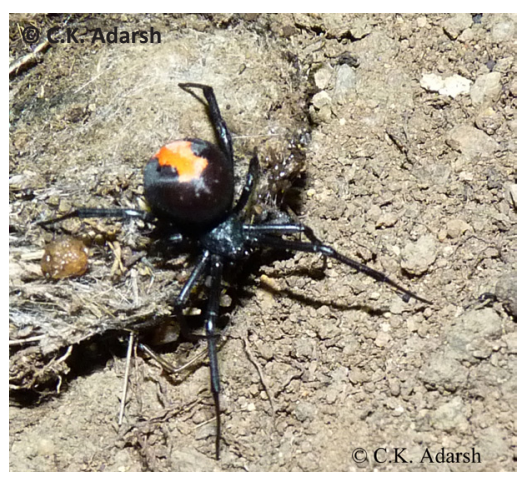

Image 56. Latrodectus hasselti

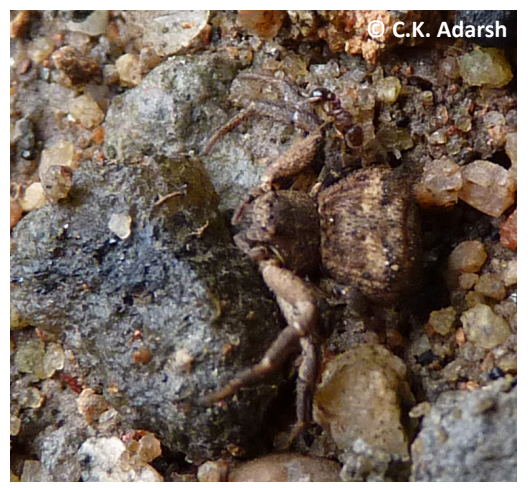

Image 59. Talaus opportunus

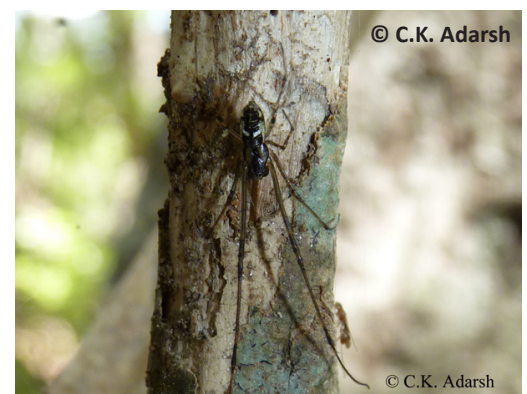

Image 51. Leucauge pondae

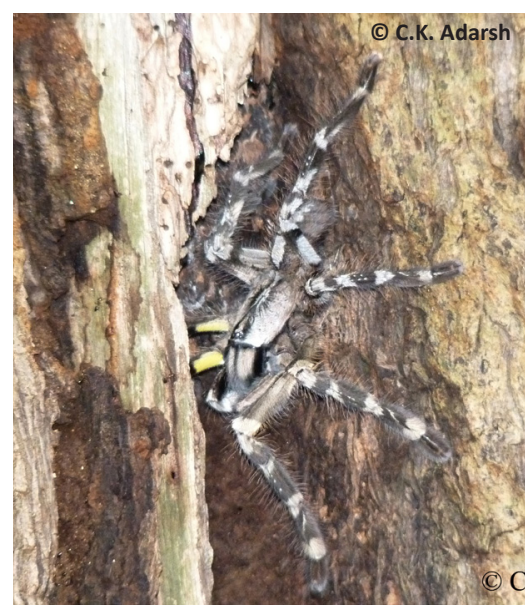

Image 54. Poecilotheria striato

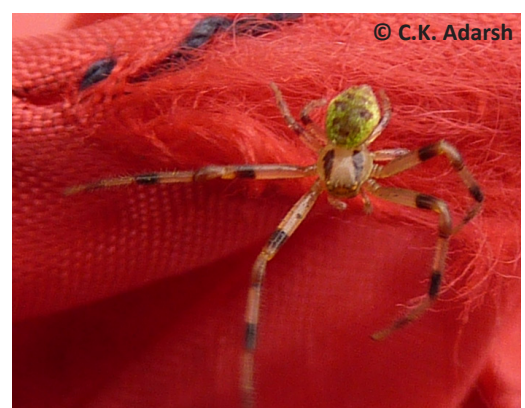

Image 57. Misumena decorata

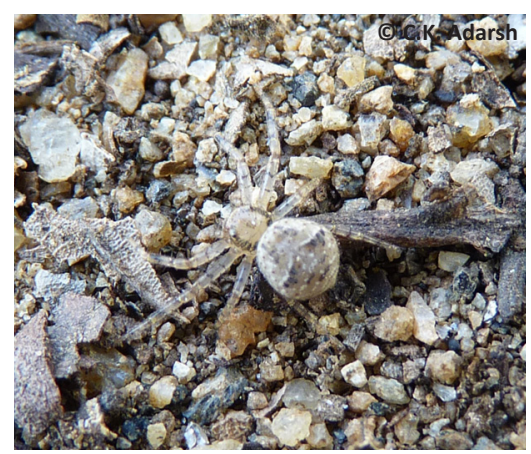

Image 60. Xysticus breviceps 


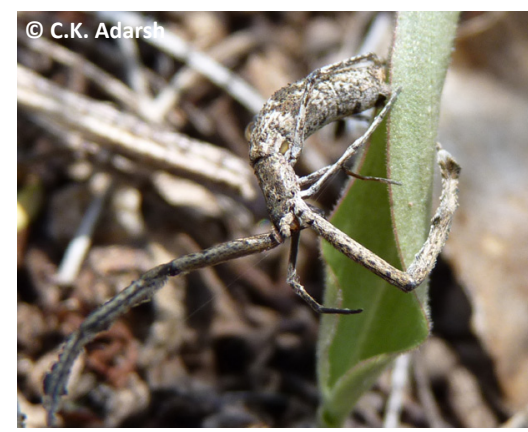

Image 61. Miagrammopes extensus

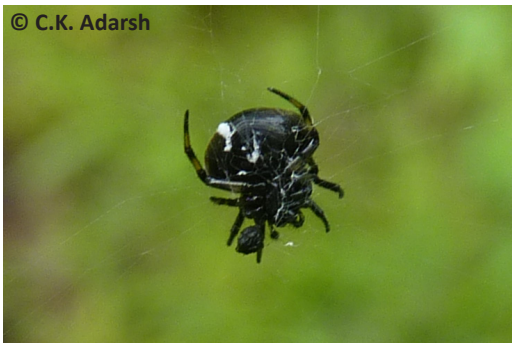

image 64. Cyrtarachne sp.

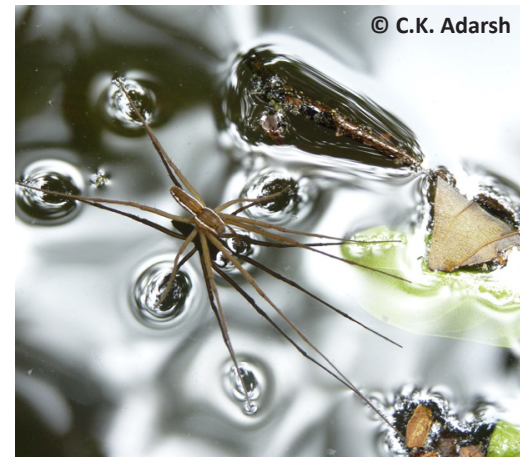

Image 67. Thalassius albocinctus

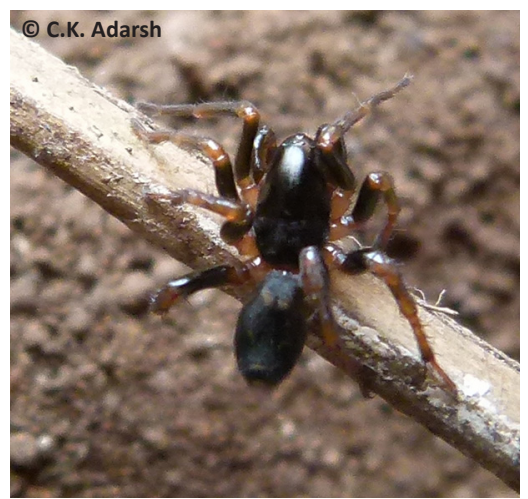

Image 62. Asceua cingulata

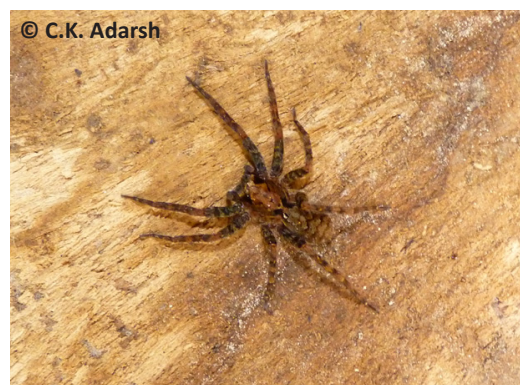

image 65. Ctenus sp.

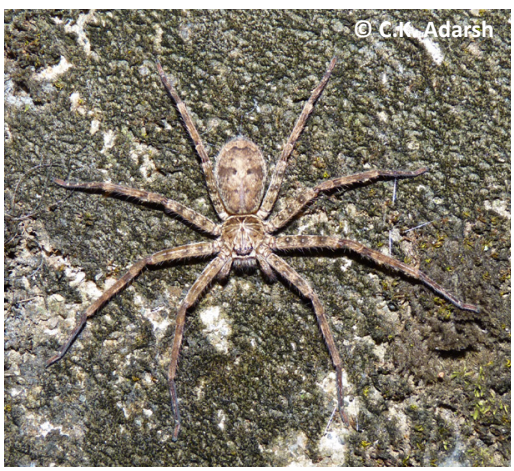

image 68. Heteropoda sp.

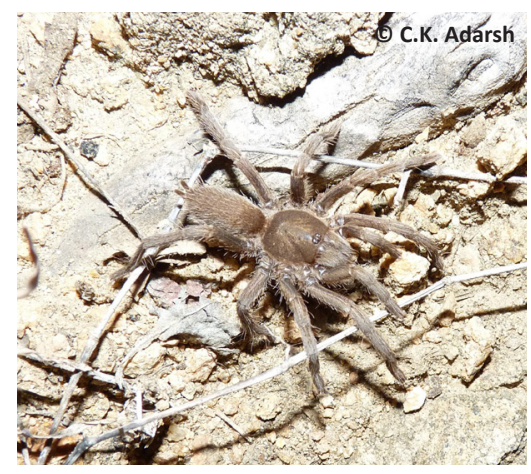

Image 63. Neoheterophrictus sp.

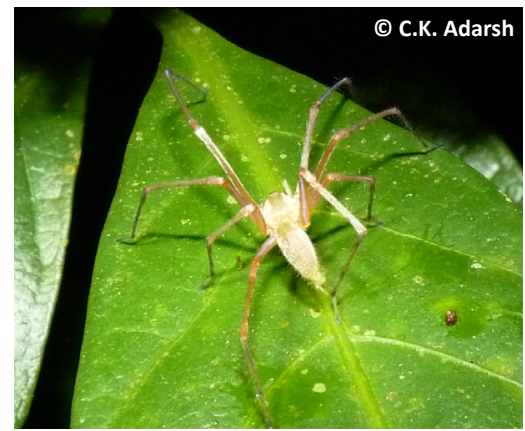

image 66. Cheiracanthium sp.

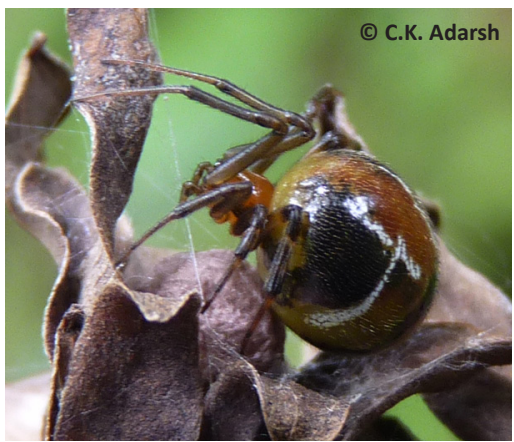

image 69. Achaearanea sp.
Subramanian, T.V. (1955). Habits and habitat of some common spiders found in Western Ghats. Journal of the Bombay Natural History Society 52 (4): 876-881.

Sudhikumar, A.V., M.J. Mathew, E. Sunish \& P.A. Sebastian (2005a). Seasonal variation in spider abundance in Kuttanadu rice agroecosystem, Kerala, India (Araneae). European Arachnology (Suppl. No. 1): 181-190.

Sudhikumar, A.V., M.J. Mathew, E. Sunish, S. Murugesan \& P.A Sebastian (2005b). Preliminary studies on the spider fauna in Mannavan shola forest, Kerala, India (Araneae). European Arachnology (Suppl. No. 1): 319-327pp.

Sugumaran, M.P., M. Ganeshkumar \& K. Ramasamy (2005). Biodiversity of spiders in Western Ghats of Tamil Nadu. Entomon 30(2): 157-163.
Tanaka, K. (1989). Movement of the spiders in arable land. Journal of Plant Protection 43: 34-39.

Tikader, B.K. (1970). Spider fauna of Sikkim. Records of the Zoological Survey of India 64: 1-83.

Tikader, B.K. (1977). Studies on spider fauna of Andaman and Nicobar islands, Indian Ocean. Records of Zoological Survey of India 72: $153-212$.

Tikader, B.K. (1980). Thomisidae (crab-spiders). Fauna of India (Araneae) 1: 1-247.

Tikader, B.K. (1982). Family Araneidae (Argiopidae), typical orb weavers. Fauna of India (Araneae) 2: 1-293.

Tikader, B.K. (1987). Handbook of Indian Spiders. Calcutta, Zoological Survey of India, 251pp.

Uetz, G.W., J. Halaj \& A.B. Cady (1999). Guild structure of spiders in major crops. Journal of Arachnology 27: 270-280.

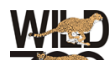

